\title{
Tangence
}

\section{Considérations con/textuelles et stratégies pédagogiques sur l'enseignement des littératures francophones de l'Afrique et des Antilles}

\section{Suzanne Crosta}

Numéro 49, décembre 1995

Les littératures francophones de l'Afrique et des Antilles

URI : https://id.erudit.org/iderudit/025879ar

DOI : https://doi.org/10.7202/025879ar

Aller au sommaire du numéro

Éditeur(s)

Tangence

ISSN

0226-9554 (imprimé)

1710-0305 (numérique)

Découvrir la revue

Citer cet article

Crosta, S. (1995). Considérations con/textuelles et stratégies pédagogiques sur l'enseignement des littératures francophones de l'Afrique et des Antilles.

Tangence, (49), 78-93. https://doi.org/10.7202/025879ar d'utilisation que vous pouvez consulter en ligne. 


\section{Considérations con/textuelles et stratégies pédagogiques sur l'enseignement des littératures francophones de l'Afrique et des Antilles}

\section{Suzanne Crosta}

Il convient plus que jamais de cultiver une lecture plurielle des cultures et des textes pour reconnaître la présence et la contribution des communautés de souche non européenne dans la constitution de divers corpus de connaissances des sciences humaines ${ }^{1}$. Une telle initiative nous engage à la tolérance et à un humanisme du dehors et du dedans de l'espace canadien. De l'aveu général, la diversité des programmes et des cours améliorent le fonctionnement de l'institution universitaire dans la mesure où elle s'ouvre sur les attentes et les désirs des communautés qu'elle dessert. Ainsi l'institution universitaire devient un lieu d'acquisition et d'actualisation pour tous ses apprenants.

Ajoutons d'autre part que l'enseignement et l'intégration des cours de littératures francophones de l'Afrique et des Antilles dans les curriculums scolaires et universitaires répondent à un véritable besoin à l'heure de la globalisation des réseaux et des marchés. La recherche, l'enseignement et la transmission des littératures (d'ici et d'ailleurs) nous ouvrent des perspectives et des pistes théoriques nouvelles tout en nous suggérant des instruments conceptuels et des définitions de problématiques qui enrichissent notre vision et notre compréhension des communautés qui constituent notre village global. L'université, en tant que lieu

1 Dans son étude, La francopbonie à l'estomac (Piris, Nouvelles du Sud, 1995, p. 87-88), Hédi Bouraoui, nous renseigne que "Parfois les immigrés récents peuvent se considérer en dépendance permanente et en équité couteuse par rapport à certains qui se disent être les seuls à posséder le privilège du facteur temps, source de légitimité irrévocable. Il serait alors amusant de parler ici de soucbitude et d'originalitude, pour dire que ce passé monopolisateur centralise pour marginaliser! [...] Il faut donc ôter cette " insécurité " qui mine de l'intérieur toutes les communautés francophones, par rapport aux pays centralisateurs, comme la France par le passé et le Québec récemment, et surtout le monoculturalisme de l'anglomanie." 
d'apprentissage et d'échanges, crée non seulement les conditions d'une socialité canadienne inclusive mais outille et prépare de futurs leaders/acteurs sociaux à bâtir des ponts et à établir des connexions dans toutes les disciplines des sciences humaines.

\section{Enseigner les littératures francophones en Ontario}

Aujourd'hui de nombreuses initiatives favorisent l'essor des littératures africaines et caribéennes. Se multiplient et s'organisent des congrès et des colloques nationaux et internationaux ayant pour axe de réflexion les littératures et les productions esthétiques de l'Afrique et des Caraïbes. Témoins de cet intérêt accru, notons les organismes (Association canadienne des études africaines, Association canadienne des études latino-américaines et caribéennes, Association des littératures africaines, Conseil international des études francophones, etc.) ainsi que les maisons d'édition (GRELCA, GREF, CIDIHCA etc.) qui travaillent activement à leur diffusion et à leur réception. En Ontario, dans le cadre institutionnel universitaire actuel, la plupart des cours de littératures africaines ou caribéennes (à quelques exceptions près) s'offrent dans des départements de littératures ou de langues européennes (anglaise, française, espagnole ou portugaise) ${ }^{2}$ ou dans le cadre des programmes spécialisés où figurent les études latino-américaines et/ou africaines. Les cours sont investis par des acteurs qui sont porteurs de projets différents et quelquefois contradictoires. Certains prônent le "Commonwealth", d'autres la "Francophonie", le "Panafricanisme", le "Postcolonialisme", le "Postmodernisme", etc. ${ }^{3}$ Les attentes des étudiants varient aussi

2 À partir de mes expériences personnelles, je peux brosser ce tableau subjectif mais peut-être pas exceptionnel de la situation des enseignants de littératures africaines et caribéennes. Je donne des cours de littérature francophone dans le Département de français, des cours particuliers de littérature africaine et caribéenne écrites ou traduites vers l'anglais dans le Programme Arts \& Science et je participe à des projets de mémoire avec le Département de langues modernes (littératures caribéennes hispanophones).

3 Pour un approfondissement des enjeux de ces diverses orientations idéologiques, on se reporte avec intêrêt aux travaux de Gerald Early, clirecteur du programme des études africaines et africaines-américaines à l'Université de Washington à St. Louis, "Understanding afrocentrism", Civilization, vol. II, $\mathrm{n}^{\circ}$ 4, 1995, p. 31-39; d'Ambroise Kom, "Francophonie et enseignement des littératures africaines: quels enjeux?" Revue francophone, vol. VIII, $\mathrm{n}^{\prime}, 1993$, p. 103-109; d'Edgar Wright, "Critical Proceclures and the Evaluation of 
80

selon leurs besoins, leurs penchants idéologiques ou leur propre sensibilité. Chacun/e arrive dans le cours portant avec lui son bagage et sa vision de ce que constituent les littératures africaines et caribéennes en matière de traditions, de modèles, de public, de valeurs, de normes, etc. Les enjeux de la réception de telle ou telle ceuvre dite "problématique" ou "hermétique" se dessinent lorsque l'arrière-fond ou le contexte échappe à la saisie du lecteur ${ }^{4}$. Les choses se compliquent encore chez les étudiants pour qui le français est une seconde langue. En effet, dans ce cas la compétence linguistique ne permet pas toujours d'apprécier les ressources de la polysémie. Il se peut en outre que les effets d'ordre émotif ne correspondent pas à leur sensibilité.

Or la décolonisation des littératures africaines et caribéennes est de plus en plus perçue comme une urgence vu les enjeux et la réception des oeuvres littéraires en dehors et au dedans du contexte de production ${ }^{5}$. Certaines contradictions se dessinent au sujet de la pédagogie des littératures africaines et caribéennes: la première étant l'inscription de l'œeuvre dans l'histoire littéraire; la deuxième, le statut de l'enseignement des littératures francophones; la troisième, les constructions sociales des représentations et projections mouvantes de l'identitaire. En ressort tout un débat sur le regard (réducteur, subjectif, objectif, etc.) de l'histoire littéraire, sur l'épistémologie où le vu et le vécu s'opposeraient au lu, sur le dialogue tantôt dynamique, tantôt polémique, tantôt impossible entre écrivains et critiques de diverses aires culturelles. Tou-

African Literature", The Critical Evaluation of African Literature (Londres, Heinemann, 1973, p. 1-22.

4 Voir l'étude d'Edward Said, Culture and Imperialism (New York, A. Knopf, 1994).

5 Dans son étude portant sur les "Problèmes méthodologiques d'une "Histoire" des Caraïbes", Jack Corzani déclare sans ambages: "Les Antilles ne sont pas des morceaux d'Afrique, pas plus qu'elles ne sont des portions de France. Ce sont des pays qui, comme d'autres, ont le droit d'être envisagés en euxmêmes, pour eux-mêmes. [...] Il est temps de décoloniser l'histoire littéraire des Caraibes et singulièrement des îles françaises, de cesser de l'élaborer depuis la métropole, tout en se gardant d'un autre impérialisme intellectuel, celui de la négritude, pleinement sensible dans la plupart des programmes des universités d'Afrique où les productions antillaises sont purement et simplement annexées, considérées comme "africaines". "Komparatistische Hefte, n 11 (1985): 54. Pour leur part, Chinweizu et al. reprochent la mentalité touristique ainsi que la "nouveaumania" qui caractérisent la démarche intellectuelle des critiques eurocentriques. Voir en plus grands détails, Toward the Decolonization of African Literature, Washington, Howard UP, 1983, p. 7-9. 
tefois, la distinction entre la littérature française et les littératures francophones engage une réflexion sur les rapports conflictuels entre le politique et le culturel ${ }^{6}$. Tout un fouillis de questions se posent et s'opposent: si les écrits francophones des Départements ou Territoires d'Outre-Mer font partie du corpus national français, pourquoi ce retard à les reconnaitre et à les promouvoir par le biais des établissements scolaires et des organismes culturels? Comment justifier ce statut d'appendice? Sur quels critères la décision d'inclure ou d'exclure tels textes du canon littéraire repose-t-elle? Il s'ensuit que ces débats se concentrent sur une certaine éthique communicationnelle du fait qu'ils entraînent des prises de position par rapport aux valeurs partagées par une communauté, sa vision, ainsi que ses manifestations et expressions culturelles.

Par ailleurs, la notion de passeport comme critère d'identification ne résout pas le problème car il ne peut opérer chez ceux pour qui l'errance est un mode d'être ou un mode de vie à l'instar d'Andrée Chédid, de Maryse Condé, d'Assia Djebar, d'Édouard Glissant, d'Abdelkébir Khatibi etc. À cela s'ajoutent les théories et les critiques de la réception, la classification ou l'identité du lectorat qui s'infiltre parfois dans les enjeux discursifs du texte et de sa critique. Certains écrivains, à l'exemple de Glissant, projettent un "lecteur futur", un lectorat virtuel ou en devenir, ne serait-ce que pour se défendre contre les reproches du lectorat actuel. On pourrait voir là un faux débat dans la mesure où il faut s'en tenir à la conscience culturelle et nationale exprimée dans les textes, que ce soit par le biais de la voix ou de la conscience de l'auteure ou des personnages mais les étanchéités thématiques et discursives sont parfois délibérément brouillées.

\section{Métaphorisation du parcours didactique}

En tant que pédagogue de la littérature en situation d'apprentissage linguistique, je considère l'analyse textuelle indispensable à la compréhension des effets multiples que peut impliquer une certaine maîtrise ou manipulation de la langue. Le travail de médiation est multiple et rempli de contradictions qu'il faut soule-

6 À cet égard, voir les sections "La francophonie sauvage", "L'écriture francophone après les indépendances" et "Langue et culture "dans La francophonie à l'estomac de Bouraoui, p. 35-41. 
82

ver et parfois assumer. Le recours à de nombreuses expérimentations dans la conception et l'élaboration des cours est inévitable pour répondre à la fois aux objectifs du pédagogue et aux attentes des étudiants. Voici quelques échantillons des méthodes et des contenus abordés: mise en valeur de la perspective diachronique/synchronique des œuvres d'écrivains africains et caribéens; exploration des tendances, des théories, des formes littéraires caractérisant les ouvres littéraires de l'Afrique et des Caraibes; parcours didactiques des textes par rapport à certaines orientations idéologiques à savoir: l'afrocentrisme, l'eurocentrisme, le féminisme, le néo/post/colonialisme, etc. Quoique ces expérimentations aient, chacune leur tour, contribué à des résultats intéressants, le principe de tracer une cartographie conceptuelle à partir de laquelle se dessinent les relations entre les métaphores ou les principes clés du cours s'est révélé d'une très grande utilité ${ }^{7}$.

Dans tous mes cours, le texte est situé par rapport aux contextes de production et de réception. L'interrogation première, c'est l'historique de la société, ses modes de connaissance et ses supports, que ce soit l'oraliture, l'écriture ou l'écran ${ }^{8}$. Il s'agit là de répondre à trois impératifs: fournir aux étudiants un point de vue subjectif ou interne du contexte en question; leur donner une vue d'ensemble qui leur permettra de percevoir les dialogues s'instaurant entre le réel et le fictif; les initier aux fils esthétiques divers de la textualité (caribéenne, marocaine, sénégalaise, tunisienne, etc.). Étant donné l'importance des traditions orales et leur influence dans les écrits francophones, l'enseignement, la recherche et la critique des textes suscitent des réflexions impor-

7 Je suis reconnaissante ici à Cheryl Amundsen de l'Université McGill dont l'atelier sur "Course Design" m'a été d'une très grande utilité pour articuler et cadrer mes pratiques et mes stratégies d'enseignement.

8 Sur les traditions orales et l'oraliture, voir les sources suivantes: Kwame Anthony Appiah, In my Fatber's House, New York, Oxford UP, 1992; Geneviève Calame-Griaule, Graines de parole, Paris, Éd. du CNRS, 1989; Ruth Finnegan, Oral Literature in Africa, Oxford, Clarendon Press, 1970; Maximilien Laroche, La double scène de la représentation, oraliture et littérature dans la Caraibe, Québec, Université Laval, GRELCA, 1991,; Wole Soyinka, Myth, Literature and the African World, Cambridge, Cambridge UP, 1976. Ma prise de position est également nourrie des réflexions des Africanistes qui ont soulevé l'urgence de valoriser l'oraliture dans la décolonisation des litteratures africaines. Chinweizu et al., Toward the Decolonization of African Literature, Washington, Howard UP, 1983, p. 1-6. 
tantes sur les limites des catégories qui opposeraient de façon globale l'oral à l'écrit sans y voir des affinités ou mêmes des continuités en ce qui concerne leurs expressions ou formes distinctives. Il y aurait également lieu d'explorer les influences esthétiques de l'activité musicale, picturale, sculpturale dans la composition et l'organisation des récits.

Pour revenir au principe de la cartographie conceptuelle ou plutôt métaphorique, celle-ci sert avant tout de point de départ pour faciliter le parcours didactique des apprenants. Il s'agit de leur faire imaginer un parcours ou même un tableau en voie de devenir. Pour aborder les contextes de production et de réception et leurs rapports avec le texte, la métaphore de deux terres fermes rattachées ou séparées (tout du dépend du regard interprétatif) par une rivière (le texte) où son cours, sa profondeur, son rythme, ses affluents, ses jeux de couleur nous renseigneraient sur la présence des éléments oraux ou littéraires du texte et par extension du corpus littéraire à l'étude. Les rapports entre la civilisation orale, la civilisation du Livre/livre, la culture et la technologie de l'œil (films, vidéos, etc.) se manifestent à divers degrés et débouchent aussi sur d'autres rivières 9 . Le texte nous invite, par le biais de l'imaginaire et de ses repères, à connaître, à apprécier cette rivière, et pour mener à bien ce projet, il faut se mouiller, et s'y jeter pour la traverser. On peut être plus ambitieux et imaginer divers ponts et là notre formation de "topos sapiens" peut être utile pour reconnaître les repères et les fondements de la rivière et des terres fermes pour poser les piliers d'un pont solide. Cette tâche est d'autant plus nécessaire que les deux contextes sont vastes et ne peuvent s'apercevoir. Il est parfois impossible de concevoir un pont et alors d'autres modes de communication doivent être cultivés pour inscrire une "poétique de relation" (expression empruntée à Édouard Glissant).

Cette cartographie conceptuelle, prise sous un angle métaphorique est conçue à des fins pédagogiques pour souligner les concepts clés: l'oralité, l'écriture, les contextes de production et de réception, les traditions et les formes particulières de l'esthétique - tous facteurs qui nous permettent de mieux

9 Le visuel (en particulier, la culture et la technologie de l'œil) figure comme lieu d'autorité d'autant plus que maintenant les jeunes et les loisirs privilégient les films, les jeux vidéos (Nintendo, Sega) ainsi que les jeux électroniques. 
84

apprécier les textes à l'étude. Elle sert avant tout de pictogramme (en mouvance) ou d'un moyen mnémotechnique par lequel l'apprenant peut traverser les textes et dans le meilleur des mondes possibles participer activement à l'élaboration de son propre tableau.

\section{Enjeux des concepts de littérature et de littérarité}

Étant donné que les textes proviennent d'espaces culturels que les étudiants ne connaissent pas à fond, l'acception de la littérature est prise dans son sens large. Elle comprend tous les écrits d'une société, d'où l'emploi des essais, des biographies, des chants, des éditoriaux, des satires, des articles de presse pour que les étudiants inscrivent le texte dans une société se trouvant à cheval sur trois modes de communication l'oral, l'écrit, et maintenant le visuel. Cette initiation ou cette insistance sur les différents systèmes de connaissances et de compétences vise à les sensibiliser aux aspects extralinguistiques comme la connaissance du monde, les stratégies de communication intrapersonnelle ou l'éthique des conventions sociales. Quant à l'appréciation esthétique, en particulier les critères de littérarité, une prise en considération des recherches précédentes effectuées dans ce domaine est indispensable. Une lecture des textes critiques ou philosophiques s'inscrivant dans une dialectique leur donne l'occasion d'identifier l'éthique ou l'idéologie derrière les prises de position de tel ou tel critique. Les reproches au sujet du caractère autobiographique/sociologique des textes africains ou caribéens, les jugements de valeur quant à la préoccupation du passé sont repris et réinterprétés en fonction des traditions philosophiques ou des conventions esthétiques du contexte à l'étude.

Peu s'en faut pour reconnaître l'apport des traditions philosophiques de pensée africaine ou de pensée arabe dans l'interprétation des textes issus de l'Afrique et des Caraibes. À titre d'exemples, les référents historiques et culturels des textes caribéens sont explicités à la lumière de l'histoire de la traite, de l'esclavage, du colonialisme, de la départementalisation et sa contrepartie les lieux et les manifestations de résistance: l'oraliture, le marronnage, la pratique du quimbois ou du vaudou, etc. Quant aux référents historiques et culturels de l'Afrique du Nord, il est essentiel de puiser dans la culture berbère, de distinguer les différentes écoles d'interprétation de l'Islam, les multiples rapports au 
sacré et au profane, la civilisation du Livre (le Coran) et l'émergence du livre comme activité séculaire, les traditions orales au féminin, etc.

\section{L'enseignement de la nouvelle de l'Afrique et des Caraïbes}

$\mathrm{Vu}$ les richesses millénaires devant lesquelles se trouve le pédagogue des littératures de l'Afrique et des Caraïbes, les critères de sélection méritent nos considérations les plus sérieuses. En guise d'exemple, un cours portant sur la nouvelle en Afrique et dans les Caraïbes ne peut se faire sans une remontée dans les traditions orales de ces contextes respectifs. En raison de leur brièveté, leur caractère ouvert et pluriel, les recueils de nouvelles correspondent à cette recherche d'un cadre, d'un sens (politique, culturel, religieux, etc.) pouvant relever du Divers tout en répondant aux contraintes temporelles d'un lectorat allant s'élargissant.

L'enseignement de la nouvelle issue des contextes des Caraïbes et de l'Afrique doit puiser dans plusieurs traditions culturelles et littéraires, d'où la nécessité de déterritorialiser les étanches discursives et narratives pour entamer des considérations relatives au tissu social, aux structures textuelles, aux figures de rhétorique (transparence opposée à opacité), aux fonctions des expressions esthétiques et langagières. Comme le fait remarquer Denise Brahimi pour la nouvelle algérienne:

[...] il y a chez certains auteurs de nouvelles une volonté explicite de se rattacher à la tradition du conte, quitte à la détourner pour d'autres fins. Cette tendance apparaît de plus en plus actuellement et permet de regrouper un certain nombre de nouvelles dans une rubrique consacrée à ce rattachement, même s'il est de pure forme et n'assure aucunement la continuité avec le conte traditionnel. ${ }^{10}$

Les rapports entre le conte et la nouvelle sont indispensables d'autant plus que les critères distinctifs entre la chanson, la fable, le conte (transcrit) et la nouvelle ne sont pas toujours opérants et la question de la littérarité reste toujours en suspens. A ce propos, il convient de souligner deux textes: Les nouveaux contes

10 Denise Brahimi, "La nouvelle algérienne", Notre Librairie, vol. 111, 1992, p. 50 . 
86

d'Amadou Koumba ${ }^{11}$ de Birago Diop et Voltaique d'Ousmane Sembène. Celui-ci puise dans les ressources et les pratiques formelles du conte alors que celui-là emprunte à ses fins la rhétorique de la nouvelle. Il est intéressant d'examiner ce dialogue entre le conte et la nouvelle pour démarquer le souci de se servir des formes usuelles de l'oralité (dialogue, style familier, apostrophe, etc.) du recours à un récit narratif centré sur un topos à l'ampleur romanesque.

On se trouve en fait en présence de formes esthétiques nouvelles pour "oraliser l'écriture". À cet égard, René Depestre avoue dans une entrevue accordée à Priska Degras et Bernard Magnier que

La nouvelle continue dans mes travaux la tradition des contes des soirées de mon enfance. J'ai tendance à lä̈ciser le conte oral qui est né dans la dépendance "cléricale" du vaudou. J'aspire à une synthèse esthétique entre le merveilleux, propre au "romancero" haïtien, et le côté toujours adorablement insolite qui entoure le quotidien-au-féminin. Mais dans la culture créole d'Haiti, outre le conte, il y a aussi la tradition picaresque de l'audience. Les Haïtiens sont des conteurs, et plus encore des audienciers-nés, c'est-à-dire des gens capables, en plein jour (car l'art du conte appartient à la nuit) de tenir en haleine, sur une galerie, un public êmerveillé, avec des histoires courtes qui, par contagion, éveilleraient le goût de la parole et du rire, même chez un arbre, un cheval ou une tortue. ${ }^{12}$

Par ailleurs, sous la rubrique "Nouvelles à entendre lire... nouvelles à entendre dire...", Claudine Richard nous renseigne sur le concept et la pratique des "diracontes", en particulier chez Francis Bebey, écrivain/conteur camerounais.

Les "diracontes" sont donc "des créations individuelles que leurs auteurs font connaître par écrit"; mais c'est en prévoyant la possibilité de pouvoir être un jour "repris oralement ou dits" que ces contes modernes deviennent des " diracontes" ${ }^{13}$

11 Diop, Birago, Les nouveaux contes d'Amadou Koumba, Paris, Présence africaine, 1967.

12 Priska Degras et Bernard Magnier, "René Depestre ou les nouvelles du géolibertin" Notre librairie, vol. 112 (1992): 85. Sur une typologie du conte dans le contexte haïtien, voir l'êtude de Maximilien Laroche dans La découverte de l'Amérique par les Américains, Québec, GRELCA, Université Laval, 1989, p. 85-105.

13 Claudine Richard; "Le renouveau d'une écriture", Notre librairie, vol. 111, 1992 , p. 21. 
Sans doute serait-il pertinent de constater dans ce procédé d'intériorisation de l'activité du conteur ou de l'activité du nouvelliste une recherche des pratiques pouvant nourrir ou rendre élastique l'expression esthétique. En ce qui concerne les Caraïbes, la nouvelle en forme de contes, en forme de "tapage oral "(terme emprunté à Ernest Pépin), serait révélatrice de ce souci de respecter ou de valoriser la "parole de nuit" (terme emprunté à Patrick Chamoiseau). Pour Ralph Ludwig, la signification des textes constituant l'éventail de cette "parole de nuit"

[...] correspond aux besoins d'une modernité en crise, d'une Europe hésitant entre la tolérance multiethnique et la nostalgie nationaliste, à un moment où les grandes idéologies s'avèrent incapables de fournir un modèle pour l'avenir. ${ }^{14}$

La nouvelle telle qu'elle se conçoit et se pratique chez les écrivain/e/s francophones de l'Afrique et des Caraibes naît et renaît dans plusieurs genres - la fable, le conte, la poésie, le roman, etc. On pourrait, en effet, y voir une sorte d'esthétique de libération ou d'ouverture pour démolir les contraintes et les diverses formes d'oppression tout en reconnaissant les complexités et les sensibilités qui correspondent aux quêtes existentielles des communautés humaines.

L'enseignement des écrits francophones se dispose à plusieurs égards à établir des dialogues transculturels visant à démolir les frontières qui séparent les êtres de leurs rapports avec euxmêmes, avec les autres et avec leur espace naturel, culturel et langagier. Un cours sur les pratiques de la nouvelle chez les écrivaines des Caraibes et de l'Afrique du Nord a débouché sur des constantes et des différences quant aux lieux et aux moments de rupture et d'ouverture avec les expressions culturelles de leur société. Le corpus comprend entre autres, Le chant des sirènes de Marie-Thérèse Colimon-Hall (Haiiti); Pays mêté suivi de Nanna$Y a$ de Maryse Condé (Guadeloupe); Femmes d'Alger dans leur appartement d'Assia Djebar (Algérie); L'oiseau de fer de Nadia Ghalem; La négresse à l'enfant de Leila Sebbar (Algérie); La planète mauve et autres nouvelles de Safia Ketou (Algérie); Femmes écbouées de Miriam Warner-Vieyra (Guadeloupe); Les corps et le temps d'Andrée Chédid (Égypte); Paroles de terre en larmes

14 Ralph Ludwig, Écrire la "parole de nuit *: La nouvelle littérature antillaise, Paris, Gallimard, 1994, p. 20. 
88

(ouvrage collectif, Guadeloupe et Martinique) et des nouvelles éparses dans différentes revues. Alors que maints regroupements peuvent se constituer en fonction d'une thématique du paysage social évoqué (contraintes politiques et culturelles, sexualisation de l'espace, éclatement de la violence et de la haïne, érotisme, etc.) ou en fonction d'une problématique esthétique particulière (l'auditif, le gestuel, le pictural), ces recueils ont en commun un certain nombre de préoccupations d'ordre éthique et esthétique.

En première analyse, le souci de dire - dire l'esclavage, l'exil, la guerre, etc. À la différence de certaines prises de position de leurs compatriotes, les écrivaines, pour la plupart, n'endossent pas la violence mais montrent son intériorisation, son refoulement (nous pensons ici à Miriam Warner-Vieyra, à Assia Djebar et à Leila Sebbar). Elles dévoilent, chacune à sa façon, les malentendus, la confusion, la peur, le désespoir, la solitude tant sur le plan individuel que sur le plan collectif. Comme nous le suggère Marie-Thérèse Colimon-Hall, dans son recueil Le Chant des sirènes, se dire en tant que femme est une tâche d'autant plus difficile qu'il faut assumer la pesanteur du réel et les responsabilités qu'impliquent tant la production que la reproduction. Dans le cadre de l'Afrique du Nord, la reconnaissance des femmes dans la lutte pour l'indépendance du pays et les souffrances encourues cèdent à une réflexion sur leur histoire personnelle et leur mise au ban de la société. Dans Femmes d'Alger dans leur appartement, Djebar se sert des pratiques du détour et du retour pour faire connaître "le murmure collectif féminin". L'organisation des nouvelles de ce dernier recueil suggère un refus de cloisonnement entre fiction et théorie d'autant plus que la "Postface" sert de réflexion, de code de lecture pour saisir la signification du texte. Femmes d'Alger dans leur appartement est traversé par des transmutations esthétiques multiples (diiwans, chants, prières, etc.) ainsi qu'une réflexion théorique sous-jacente. Bref, il est possible de relever chez Colimon-Hall comme chez Djebar plusieurs stratégies pour valoriser la polyphonie et la texture polysémique des récits qui cherchent à poser les jalons d'une parole tantôt en gestation tantôt en mouvance.

En deuxième analyse, le rapport difficile avec l'(H)histoire qui se voile et se dévoile dans les recueils de nouvelles s'accompagne d'une volonté de tendre vers l'autre. Les nouvelles de Pineau ou de Colimon-Hall contiennent des récits où les narratrices écrivent l'amour, les moments de rupture comme les 
moments de déchirure. D'un ton plus angoissant, le recueil de nouvelles de Warner-Vieyra parle de l'amour, dans sa difficulté à être dans "Le Mur ou les charmes d'une vie conjugale", et dans son potentiel à la violence à l'exemple de la scène de castration dans "Sidonie". Assia Djebar, Leila Sebbar, Nadia Ghalem évoqueront, chacune, la sexualisation de l'espace et en l'absence de communication entre hommes et femmes, la répression et l'enfermement physique et affective des femmes. Le recueil de nouvelles rassemble les fragments de l'existence, les bribes de voix délaissées ou étouffées et ainsi jette lumière sur le refoulé et le désir de se réconcilier avec soi et avec l'autre. C'est Sarah dans Femmes d'Alger dans leur appartement qui tracera une voie thérapeutique: "Je ne vois pour les femmes arabes qu'un seul moyen de tout débloquer: parler, parler sans cesse d'hier et d'aujourd'hui, parler entre nous, dans tous les gynécées, les traditionnels et ceux des H.L.M." ${ }^{15}$.

En troisième analyse, le recours à l'épistolaire n'est pas à négliger dans les nouvelles des écrivaines de l'Afrique et des Caraibes. Dans le recueil, Chant des sirènes, la lettre s'insinue de différentes façons. Presque toutes les protagonistes ont accès à la lettre; non seulement elles en écrivent, mais aussi elles en reçoivent. Le plus souvent, cependant, elles attendent impatiemment le courrier qui ne vient jamais remplir leur vou. Dresser une typologie de lettres que l'on retrouve dans le recueil de ColimonHall et leurs fonctions dans le recueil nous permet de dégager certains principes qui structurent le recueil (lettre d'aveu, lettre officielle, lettre de rupture, billets doux, etc.). Le repérage d'éléments formels (nombre de lettres contenues, extraits ou intégralité de la lettre, localisation et datation, signature ou destinataire) offrent à l'apprenant une saisie des conventions sociales et littéraires eu égard au statut et aux fonctions de la lettre. Le souci des dates chez Colimon-Hall et de l'identité de la destinataire marquent à quel point le référent pèse sur les êtres. Le parcours et la modalité de la lettre sont aussi à relever: lecture (de la lettre intégrale ou quelques extraits), traduction, réception (réactions affectives et esthétiques). L'importance de la voix narrative est à souligner, car il s'agit de suivre l'itinéraire et la constitution parcellaire mais progressive de l'identitaire du sujet au féminin. Il est signifi-

15 Assia Djebar, Femmes d'Alger dans leur appartement, Paris, Des Femmes, 1980, p. 68. 
90

catif de noter que la lettre dans les recueils des écrivains de l'Afrique du Nord à l'instar d'Assia Djebar ou de Tahar Ben Jelloun doit passer par la traduction de l'arabe. L'activité traductrice se transforme en activité créatrice du fait qu'elle s'inscrit dans la thématique et la symbolique du récit.

En dernière analyse, notons les recueils à facture symbolique tels que La négresse à l'enfant de Sebbar, Le corps et le temps d'Andrée Chédid, L'oiseau de fer de Nadia Ghalem, La planète mauve et autres nouvelles de Safia Ketou, qui nous laissent entrevoir cette notion d'ouverture à partir de regards multiples sur l'univers. Se dire en symboles, se submerger, s'envoler ou errer dans des espaces mouvants suggère un certain désir d'outrepasser les frontières, les murs, les catégorisations qui figent l'être et son avenir. Il n'est donc pas surprenant que maintes écrivaines abordent la question de l'émigration, la possibilité ou l'impossibilité de s'intégrer dans le paysage social évoqué, la recherche d'identité, l'affirmation d'un lieu entre ici et ailleurs et dont le devenir est toujours à imaginer et à espérer. L'esthétique nouvellière (le rapport cadre/nouvelles) s'apprête à l'exploration des héritages et des confessions multiples qui rendent mouvants les critères rigides d'une identité nationale.

\section{Pratiques et stratégies pédagogiques}

Étant donné que le corps estudiantin se compose, pour la plupart, d'anglophones, monter un cours sur la nouvelle est stratégique puisque la longueur ne les intimide pas. Les apprenants sont encouragés à creuser le texte et à analyser les structures de pensée qui sous-tendent l'expression. S'il y a eu dans mes pratiques d'enseignement un glissement d'intérêt vers les modes interactionnels, le dessein est de permettre aux apprenants de participer activement à leur apprentissage. Celui-ci ne peut que se faire à double sens dans la mesure où on apprend à dialoguer en bonne entente (reconnaissance et non dissolution des différences). Il est certainement vrai qu'une connaissance préalable du référentiel est plus importante que la complexité syntactique du texte puisque l'apprenant a déjà une saisie de ce contre quoi réagit l'auteur/e. Pour cette raison, il est indispensable de fournir aux étudiants des lectures critiques de pointe qui contribuent à stimuler la discussion sur la littérature et ses rapports avec d'autres disciplines à l'exemple du politique, du sociologique et 
du culturel ${ }^{16}$. Comme je l'ai déjà signalé au début de ce travail, il est aussi essentiel de questionner les deux terrains d'enquêtes: le contexte de production et le contexte de réception quant aux facteurs de race, classe, sexe, culture, âge et mobilité (habilité) ${ }^{17}$. La prise de vue et de parole des écrivains enrichit leur aptitude de lecture des textes francophones grâce à la reconnaissance des expressions de résistance aux réductions, aux simplifications, aux cloisonnements et aux censures de toutes sortes.

Dans un premier temps, les objectifs d'apprentissage comprennent nécessairement l'analyse textuelle en fonction des traits constitutifs de la nouvelle (focalisation, voix, caractérisation, temps, espace, thèmes, etc.) et en fonction des procédés littéraires, en particulier les figures de style jouant sur le signe, le signifié, le signifiant, l'ordre des mots, ainsi que leur mise en présence dans le texte ${ }^{18}$. Mais dans un deuxième temps, les objectifs peuvent s'élargir pour:

- montrer les conditions et les contraintes se posant, s'imposant, s'opposant aux écrivains tour à tour dans chacun des contextes à l'étude

- initier l'apprenant à différents types de lectures de décentrement ou de recentrement de l'histoire littéraire où se manifestent des mouvements d'affirmation et de transfiguration, entre autres, la Renaissance Harlem, I'Indigénisme haïtien, la Négritude, l'antillanité, la créolité, théories et discours sur le néo/post/colonialisme, etc.;

- préciser les outils d'alphabétisation et le rapport à l'écriture ;

- souligner les conditions de la langue d'écriture et le rapport à la littérature à la lumière des contraintes

16 À ce sujet; l'initiative de GRELCA de publier chaque année les parutions les plus récentes sur la littérature haïtienne est un modèle à suivre pour donner aux étudiants les outils et l'information dont elle/il aura besoin pour actualiser et approfondir ses connaissances. Voir à titre d'exemple, Bibliographie d'Haiti sous la direction de Maximilien Laroche, Québec/Haiti, Université Laval/Université Quisqueya, 1995. Aussi faudra-t-il aussi inclure les réseaux électroniques disponibles dans la diffusion des connaissances.

17 L'étude de Greta Nemiroff est utile à cet égard. Voir le chapitre intitulé "Reconstructing Pedagogy" dans Reconstructing Education: Toward a Pedagogy of Critical Humanism, Toronto, OISE Press, 1992, p. 75-90.

18 Voir à très grande utilité l'étude de Christine Klein-Lataud, Précis des figures de style, Toronto, Éd. du GREF, 1991. 
(graphiques, syntactiques, sémantiques, contextuelles...) dans la production du sens du récit;

- relever les stratégies et/ou subversions du genre littéraire et du positionnement de l'écrivain/e par rapport aux facteurs de race, de classe, de sexe, etc. et, le cas échéant, leur transfiguration ou leur dépassement.

$\mathrm{Si}$, autrefois, il s'agissait d'initier l'apprenant aux textes-témoignages relatant les expériences des communautés francophones (et là tout est relatif), maintenant la composition du corps estudiantin nous permet d'entrer en dialogue avec les expériences personnelles des membres des communautés culturelles en question. Au fond, il s'agit de reconnaître les champs d'affectivité qui jouent sur les horizons significatifs du texte. Un exercice de lecture-écriture permet aux apprenants de prendre conscience des représentations de différents types de nouvelles et de leurs principes organisationnels. L'exercice comprend la rédaction d'un texte selon l'interprétation active et rétrospective du lecteur devenu à son tour nouvelliste, des choix qu'il/elle opérera et des effets qu'il/elle aimerait produire. Par ailleurs, la variété des stratégies suscite l'intérêt des étudiants. Quoique j'aie adopté pour le moment la stratégie des mini-conférences (15-20 minutes), j'adopte par la suite des ateliers ou des activités où l'interaction est de mise (commentaires, impressions, rédaction d'une minute sur une question, l'entrée du journal, etc.). Le travail sous forme d'atelier (les cellules d'apprentissage) combiné avec les mini-conférences est la meilleure formule jusqu'à présent pour répondre aux besoins particuliers de mes apprenants.

\section{En guise de conclusion}

L'enseignement des littératures (de quelque aire culturelle qu'elle provienne) est inextricablement liée à notre vision du monde que maints théoriciens et praticiens de la pédagogie nous recommandent d'élargir pour inclure autant de voix et de perspectives différentes ${ }^{19}$. Ainsi, l'apprentissage se fait à double sens

19 Pour une discussion et une analyse approfondie d'une pédagogie "libératrice" ou "transgressive", je renvoie les lecteurs aux travaux suivants: Paulo Freire, The Politics of Liberation, Massachusetts, Bergin \& Garvey Publishers, 1985; Greta Hofmann Nemiroff, Recontructing Education, Toronto, OISE 
dans la mesure où on enseigne à réfléchir de façon critique, à remettre en question les prémisses et les assises de nos connaissances et nos modes de transmission, à identifier les stéréotypes et les préjugés qui s'infiltrent dans nos discours, à déconstruire les mythes et les mystifications populaires d'antan et de notre époque. Voilà un investissement collectif qui mérite bien d'être relevé. 